INFLAMMATORY BOWEL DISEASE

\title{
The RANKL/OPG system is activated in inflammatory bowel disease and relates to the state of bone loss
}

\author{
A R Moschen, A Kaser, B Enrich, O Ludwiczek, M Gabriel, P Obrist, A M Wolf, H Tilg
}

Gut 2005;54:479-487. doi: 10.1136/gut.2004.044370

See end of article for authors' affiliations

Correspondence to: Dr H Tilg, Department of Medicine, University Hospital Innsbruck, Anichstr 35, 6020 Innsbruck, Austria; Herbert.Tilg@uibk.ac.at

Revised version received 13 September 2004 Accepted for publication 21 September 2004

\begin{abstract}
Background and aims: A substantial proportion of patients with inflammatory bowel disease (IBD) develops osteopenia and osteoporosis in the course of disease. Recent data from a mouse model of colitis suggest that the receptor activator of nuclear factor kappa B (RANKL)/osteoprotegerin (OPG) system may be responsible for bone loss.

Methods: We investigated the activation state of the RANKL/OPG system and its association with bone loss in human IBD. Plasma levels of OPG and RANKL were correlated with bone mineral density and current IBD therapy. Colonic secretion of OPG and RANKL and cell types responsible for such secretion were determined.

Results: OPG plasma levels were elevated 2.4-fold in Crohn's disease (CD) and 1.9-fold in ulcerative colitis (UC) whereas soluble RANKL (sRANKL) levels were not significantly different in IBD patients compared with healthy controls. High levels of OPG were released from colonic explant cultures (CEC) derived from inflamed IBD specimens, and colonic macrophages and dendritic cells costained for OPG. sRANKL levels from CEC were low both in IBD patients and healthy controls. Interestingly, increased expression of RANKL was mainly confined to cells in the lamina muscularis. A significant negative correlation was found between OPG plasma levels and femoral neck/lumbar spine bone mineral density. Conclusions: We have demonstrated that IBD is associated with alterations in the RANKL/OPG system. Applying results from a murine model of colitis associated bone loss, the constellation of OPG and sRANKL regulation observed in our study raises the possibility that RANKL/OPG may contribute to the development of bone loss in IBD.
\end{abstract} gerin $(\mathrm{OPG}) .^{13}{ }^{14}$ Activated $\mathrm{T}$ cells produce RANKL and its soluble form, sRANKL, and can directly trigger osteoclastogenesis and bone loss through its receptor RANK. ${ }^{13}$ Ashcroft et al have recently demonstrated that in IL-2 deficient colitic mice, bone loss is mediated via activated $\mathrm{T}$ cells secreting RANKL. ${ }^{10}$ Although transcription as well as plasma levels of the decoy receptor of RANKL, OPG, are substantially increased in IL-2 deficient mice, this upregulation of OPG does not suffice to ameliorate bone loss by $\mathrm{T}$ cell derived RANKL while, more importantly, exogenous administration of OPG to IL-2 deficient mice reverses bone loss, and notably also ameliorates intestinal inflammation. ${ }^{10}$

Given that activated $\mathrm{T}$ cells are central in human IBD, we hypothesised that inflammation per se via the RANK/ RANKL/OPG pathway could contribute to bone loss in IBD. Indeed, we found that this system is activated in CD and UC, and that OPG plasma levels inversely correlate with bone mineral density.

\section{PATIENTS AND METHODS \\ Patient population}

A total of 180 consecutive patients with an established diagnosis of IBD (111 CD and 69 UC) were involved in the study, with patients either contributing plasma or colon mucosa samples to the study. The control group was recruited from 75 age and sex matched healthy individuals. Baseline characteristics of the patients are shown in table 1 . Informed consent was obtained from each patient involved in the study. Plasma samples were obtained from 112 patients and 45 healthy controls. Blood $(9 \mathrm{ml})$ was collected into Sarstedt Monovette EDTA containers, centrifuged at $1200 \mathrm{~g}$ for 15 minutes at $4^{\circ} \mathrm{C}$, aliquoted into $1 \mathrm{ml}$ portions, and stored at $-80^{\circ} \mathrm{C}$ until assay.

Abbreviations: $C D$, Crohn's disease; UC, ulcerative colitis; IBD, inflammatory bowel disease; OPG, osteoprotegerin; RANKL, receptor activator of nuclear factor kappa B; sRANKL, soluble receptor activator of nuclear factor kappa $B ; B M D$, bone mineral density; $m A b$, monoclonal antibody; $\mathrm{pAb}$, polyclonal antibody; $\mathrm{CEC}$, colonic explant culture; TNF, tumour necrosis factor; IL, interleukin; ELISA, enzyme linked immunosorbent assay 


\begin{tabular}{|c|c|c|c|}
\hline & Controls & $\begin{array}{l}\text { Crohn's } \\
\text { disease }\end{array}$ & $\begin{array}{l}\text { Ulcerative } \\
\text { colitis }\end{array}$ \\
\hline $\mathrm{n}$ & 75 & 111 & 69 \\
\hline Age $(y)^{*}$ & $\begin{array}{l}36.8(14.5) \\
{[18.2-78.8]}\end{array}$ & $\begin{array}{l}33.4(13.6) \\
{[19.1-75.8]}\end{array}$ & $\begin{array}{l}36.4(12.4) \\
{[18.8-64.7]}\end{array}$ \\
\hline $\operatorname{Sex}(M / F)$ & $33 / 42$ & $50 / 61$ & $36 / 33$ \\
\hline Disease duration $(y)^{*}$ & & $\begin{array}{l}3.12(4.93) \\
{[0-23.3]}\end{array}$ & $\begin{array}{l}4.69(6.8) \\
{[0-23.8]}\end{array}$ \\
\hline Activity score (mean (SD) & & $257(67) \dagger$ & $7.7(2.1) \ddagger$ \\
\hline \multicolumn{4}{|l|}{ Medication } \\
\hline None & 75 & 25 & 10 \\
\hline Aminosalicylate & & 46 & 36 \\
\hline Systemic steroid & & 30 & 21 \\
\hline Topical steroid & & 4 & 2 \\
\hline Azathioprine & & 12 & 2 \\
\hline Antibiotic & & 11 & - \\
\hline Ciclosporine & & 1 & 1 \\
\hline \multicolumn{4}{|c|}{$\begin{array}{l}\text { "Values are mean (SD) [range]. } \\
\text { †Crohn's disease activity index (CDAl) }{ }^{25} \\
\text { †Activity assessed according to Rachmilewitz. } \\
\text { "Some patients had combined therapy. }\end{array}$} \\
\hline
\end{tabular}

\section{Colonic explant cultures (CEC)}

Involved and non-involved colonic biopsies were obtained from a group of 68 IBD patients, 40 with CD and 28 with UC. The colonic mucosa was first assessed by gross endoscopic appearance and further specified by histological evaluation of biopsies taken in parallel. Thirty patients undergoing screening colonoscopy served as the healthy control population. CEC were performed essentially as described previously ${ }^{15}$ with minor modifications. Briefly, colonic biopsy specimens were collected in phosphate buffered saline $\left(4^{\circ} \mathrm{C}\right)$, washed, weighed, and cultured in duplicate for 24 hours in 48 well plates in $1 \mathrm{ml}$ of RPMI 1640 (Schoeller Pharma, Vienna, Austria) supplemented with $10 \%$ heat inactivated $\left(56^{\circ} \mathrm{C}\right.$, 30 minutes) fetal calf serum, $100 \mathrm{U} / \mathrm{ml}$ penicillin $\mathrm{G}$, and $10 \mu \mathrm{g} / \mathrm{ml}$ streptomycin (all Gibco BRL, Vienna, Austria). Subsequently, the supernatant was collected and stored at $-80^{\circ} \mathrm{C}$ until measurement of OPG and sRANKL levels.

\section{OPG and sRANKL enzyme linked immunosorbent assay (ELISA)}

Levels of OPG and sRANKL in plasma specimens and supernatants of CEC were determined by commercially

Table 2 Bone mineral density in the inflammatory bowel disease study population

\begin{tabular}{ll}
\hline Patient characteristic & \\
\hline $\mathrm{n}$ & 43 \\
Crohn's disease (n) & 29 \\
Ulcerative colitis (n) $^{*}$ & 14 \\
Age (y) & $33.14(1.12)[19.2-59.2]$ \\
Disease duration (y)* & $3.4(5.7)[0.2-23.7]$ \\
Time (y) between OPG/sRANKL & $0.4(0.4)[0.0-1.1]$ \\
$\quad$ measurement and BMD assessment & \\
OPG (pg/ml)* & $1986.13(751.53)$ \\
& {$[963.06-3983.19]$} \\
sRANKL (pg/ml)* & $7.83(4.51)[2.9-28.2]$ \\
OPG/sRANKL* & $292.44(21.35)$ \\
& {$[110.69-632.77]$} \\
BMD (g/cm ${ }^{2}$ ) lumbar vertebrae & $0.912(0.15)$ \\
T score (L1-L4) & $-1.4(1.48)$ \\
Z score (L1-L4) & $-1.25(1.46)$ \\
BMD (g/cm ${ }^{*}$ ) femoral neck & $0.713(0.12)$ \\
T score (femoral neck) & $-1.5(1.13)$ \\
Z score (femoral neck) & $-1.2(1.17)$ \\
\hline
\end{tabular}

*Values are mean (SD) [range].

OPG, osteoprotegerin; RANKL, receptor activator of nuclear factor kappa B; sRANKL, soluble receptor activator of nuclear factor kappa B; $B M D$, bone mineral density. available specific ELISAs according to the manufacturers' protocols (DuoSet Development System, human OPG/ TNFRSF11B, R\&D Systems, Minneapolis, Minnesota, USA; sRANKL ELISA, Biomedica GmbH, Vienna, Austria). The lower detection limit of the OPG assay was $62.5 \mathrm{pg} / \mathrm{ml}$ and that of sRANKL $1.6 \mathrm{pg} / \mathrm{ml}$. Absorption was determined with an ELISA reader at $450 \mathrm{~nm}$.

\section{Biochemical parameters and bone mineral density (BMD) assessment}

Testing for routine biochemical parameters was performed in the central laboratory of the University Hospital Innsbruck according to standard automated techniques. BMD was assessed with a Hologic QDR 4500W $x$ ray bone densitometer for PA lumbar spine and proximal femur (femoral neck) in a subpopulation (total 43 IBD patients) of those patients that participated in the plasma levels study. BMD was performed on clinical grounds (that is, where indicated on a periodic schedule as part of the routine management of our IBD patients). BMD, and $\mathrm{T}$ and $\mathrm{Z}$ scores were determined for each patient according to the WHO Study Group Recommendations for the Definition of Osteoporosis and Osteopenia ${ }^{16}$ : a T score of -1 to -2.5 was classified as osteopenia and a $\mathrm{T}$ score $\leqslant-2.5$ was classified as osteoporosis. Details of the patients assessed are presented in table 2 .

\section{Immunohistochemistry}

Sections of colonic tissue specimens $(4 \mu \mathrm{m})$ were prepared from formalin fixed paraffin embedded specimens of colonic biopsies. Additionally, several historical surgical resection specimens obtained from the Institute of Pathology were also included in this analysis. Sections were deparaffinised in xylene and rehydrated in graded alcohols. A high temperature antigen retrieval technique by autoclaving slides in antigen unmasking solution (Vector Laboratories, Burlingame, California, USA) was used for OPG detection. For RANKL detection, antigen retrieval was performed by proteolytic treatment with proteinase $\mathrm{K}$ (DakoCytomation, Glostrup, Denmark). Endogenous peroxidase activity was quenched with $3 \% \mathrm{H}_{2} \mathrm{O}_{2}$ in phosphate buffered saline for 10 minutes. Endogenous alkaline phosphatase activity was blocked with levamisole solution. Non-specific binding sites were blocked with serum free protein block (DakoCytomation) for 10 minutes. For OPG detection, a mouse antihuman OPG monoclonal antibody (mAb) was purchased from Imgenex (San Diego, California, USA). RANKL was detected by a mouse antihuman RANKL mAb from R\&D Systems. Appropriate control antibodies for mouse $\operatorname{IgG}_{1}$ and mouse $\operatorname{IgG}_{2 \mathrm{~b}}$ were purchased from DakoCytomation. Slides were incubated with primary antiRANKL $(1: 25 ; 20 \mu \mathrm{g} / \mathrm{ml})$, anti-OPG $(1: 50 ; 10 \mu \mathrm{g} / \mathrm{ml})$, and control Abs, diluted in Dako antibody diluent at $4^{\circ} \mathrm{C}$ overnight. After washing $(3 \times 5$ minutes in phosphate buffered saline) OPG was visualised with a Dako LSAB+ Kit (DakoCytomation) with AEC (3-amino-9-ethylcarbazol) substrate and RANKL using a Vectastain ABC alkaline phosphatase kit with Vector Red alkaline phosphatase substrate (Vector Laboratories). Finally, sections were counterstained with haematoxylin QS (Vector Laboratories). OPG slides were aqueously mounted in Dako Faramount Mounting Medium and RANKL slides were dehydrated and mounted permanently in Eukitt ( $\mathrm{O}$ Kindler GmbH, Freiburg, Germany).

\section{Double immunofluorescence labelling}

Colonic tissue specimens were embedded in Tissue-Tek (Sakura Finetek Europe, Zoeterwoude, Netherlands). Sections ( 5 and $10 \mu \mathrm{m}$ ) were prepared, dried overnight, and fixed in $4^{\circ} \mathrm{C}$ acetone. For all staining experiments, isotype matched control antibodies were included to exclude 

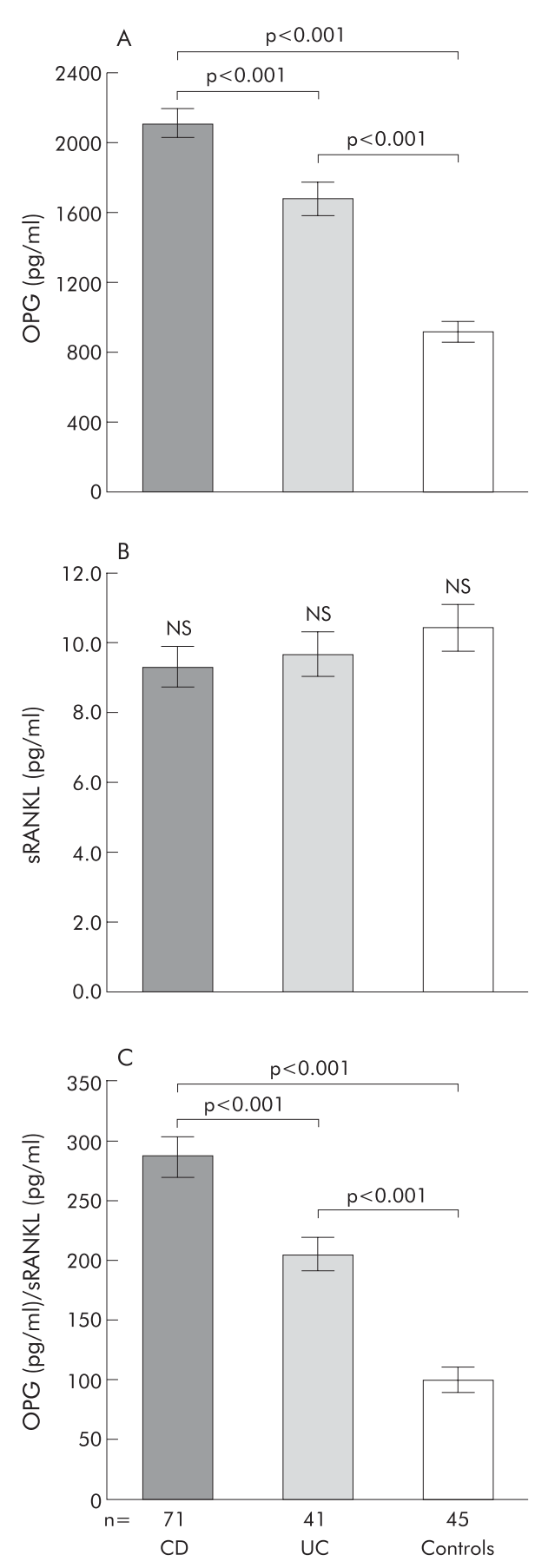

Figure 1 Plasma levels of osteoprotegerin (OPG) (A), soluble receptor activator of nuclear factor kappa $B$ (sRANKL) (B), and the OPG/sRANKL ratio $(C)$. OPG and sRANKL plasma concentrations were determined in patients with Crohn's disease (CD) and ulcerative colitis (UC) and depicted either as a single value or as OPG/sRANKL ratio compared with healthy controls.

non-specific staining. Sections were stained with anti-OPG pAb (Santa Cruz) and anti-RANKL mAb (R\&D Systems) in phosphate buffered saline and $1 \%$ bovine serum albumin at $4^{\circ} \mathrm{C}$ overnight. After washing, OPG and RANKL were visualised by incubating with secondary biotinylated antirabbit and antimouse Ig (1:200) followed by streptavidinTexas Red (1:200) (all Vector Laboratories). Subsequently, sections were incubated with anti-CD3 (BD Biosciences, San Jose, California, USA), anti-MHC II (DakoCytomation), antiCD163 (BMA Biomedicals, Augst, Switzerland), and anti-DC-
Table 3 Correlation of biochemical parameters with osteoprotegerin (OPG) and soluble receptor activator of nuclear factor kappa B (sRANKL)

\begin{tabular}{lll}
\hline & OPG $(\mathrm{pg} / \mathrm{ml})$ & sRANKL $(\mathrm{pg} / \mathrm{ml})$ \\
\hline OPG (pg/ml) & & $r=0,244 ; \mathrm{p}<0.001$ \\
sRANKL (pg/ml) & $r=0.244 ; \mathrm{p}<0,001$ & $\mathrm{NS}$ \\
Blood sedimentation rate & $\mathrm{NS}$ & $\mathrm{NS}$ \\
Leucocytes & $r=0.159 ; \mathrm{p}<0.05$ & $\mathrm{NS}$ \\
Erythrocytes & $r=-0.270 ; \mathrm{p}<0.001$ & $\mathrm{NS}$ \\
Haemoglobin & $r=-0.233 ; \mathrm{p}<0.001$ & $\mathrm{NS}$ \\
Haematocrit & $\mathrm{NS}$ & $\mathrm{NS}$ \\
Platelets & $r=0.177 ; \mathrm{p}<0.05$ & $r=-0.214 ; \mathrm{p}<0.01$ \\
Transferrin & $\mathrm{NS}$ & $\mathrm{NS}$ \\
Ferritin & $\mathrm{NS}$ & $r=0.363 ; \mathrm{p}<0.001$ \\
C reactive protein & $\mathrm{NS}$ & $\mathrm{NS}$ \\
$\alpha_{1}$ Glycoprotein & $\mathrm{NS}$ & $\mathrm{NS}$ \\
\hline & & \\
\hline
\end{tabular}

LAMP (BeckmanCoulter, Fullerton, California, USA) in phosphate buffered saline and $1 \%$ bovine serum albumin for one hour at room temperature. This step was followed by an isotype specific antimouse $\mathrm{IgG}_{1}$ Alexa fluor 488 conjugated Ab (Molecular Probes Inc., Eugene, Oregon, USA). Nuclear counterstaining was performed with DAPI $\left(4^{\prime}, 6^{\prime}-\right.$ diamidino-2-phenylindole) in phosphate buffered saline (1:1000) for 12 minutes (Vysis, Downers Grove, Illinois, USA). Finally, sections were mounted in Dako fluorescent mounting medium. Sections were viewed on a Carl Zeiss Axiovert 200M epifluorescence microscope with AxioCam and AxioVision software.

\section{Statistical analysis}

Statistical analysis was performed with the SPSS 11.0 software package (SPSS, Chicago, Illinois, USA) with ANOVA followed by the post hoc Bonferroni test. Differences were considered significant at $p<0.05$. The relationships between OPG, RANKL, and other parameters were determined using the Pearson correlation technique.

\section{RESULTS}

\section{OPG plasma levels are increased in IBD}

We assessed OPG and sRANKL plasma levels in 112 patients with IBD and 45 control subjects. As depicted in fig lA, plasma levels of OPG were significantly increased in CD as well as in UC. Highest OPG levels were detected in CD (CD 2111 (83) pg/ml, control 867 (48) pg/ml; p<0.001) (fig lA). In IBD patients, OPG plasma levels were weakly positively correlated with leucocyte and platelet counts, while a negative correlation was found for erythrocyte counts (table 3).

In contrast, sRANKL plasma levels were not significantly different in IBD patients compared with healthy control subjects, and there were no differences between CD and UC (fig 1B). It should be noted that our detection system primarily detects free unbound sRANKL, thus not complexed with OPG and therefore presumably biological active.

To gain further insight into the activation state of the OPG/ RANKL system, we calculated the OPG/SRANKL ratio based on the plasma level data. As outlined in fig $\mathrm{lC}$, the OPG/ sRANKL ratio was significantly higher in $C D$ and UC compared with control subjects, with CD showing the highest OPG/sRANKL ratio (CD 286.2 (16.6); control 98.1 (8.3); $\mathrm{p}<0.001)$.

\section{Osteoprotegerin plasma levels are inversely correlated with BMD}

Next, we assessed the relationship between OPG plasma levels and BMD, particularly in IBD patients. A significant 

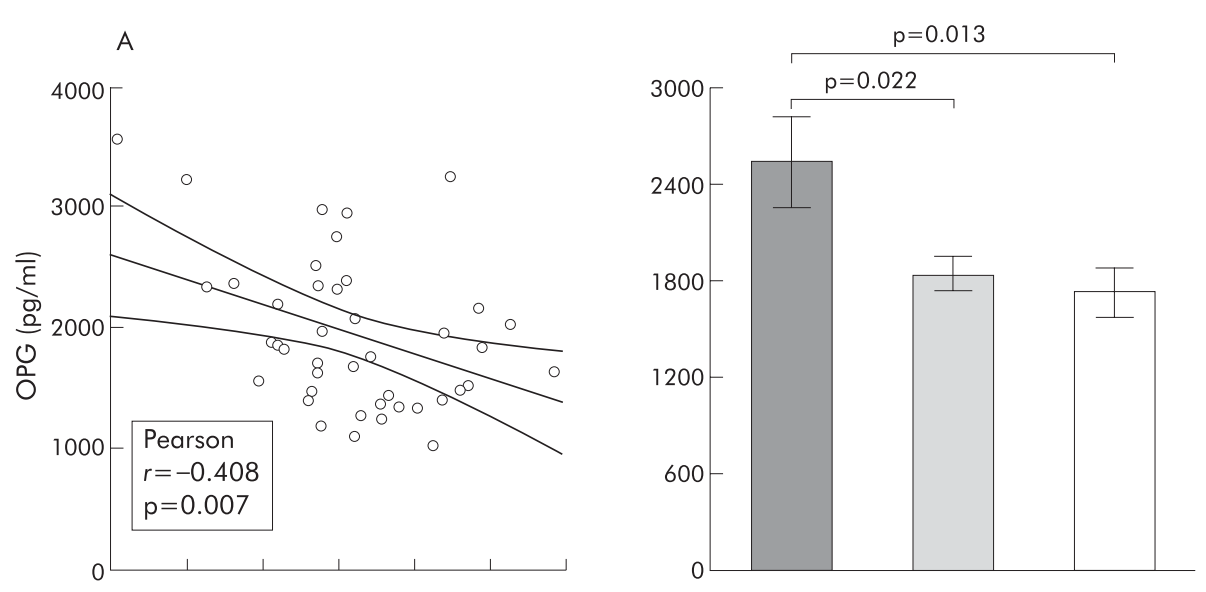

Figure 2 Osteoprotegerin (OPG) and soluble receptor activator of nuclear factor kappa B (sRANKL) plasma levels and bone mineral density (BMD) in inflammatory bowel disease (IBD) patients. Left panels show the correlation of OPG levels (A), sRANKL plasma levels (B), and the OPG/ sRANKL ratio $(C)$ with femoral neck BMD. Right panels show analysis of OPG, sRANKL plasma levels, and the OPG/sRANKL ratio in IBD patients classified as normal, osteopenic, and osteoporotic according to their BMD.
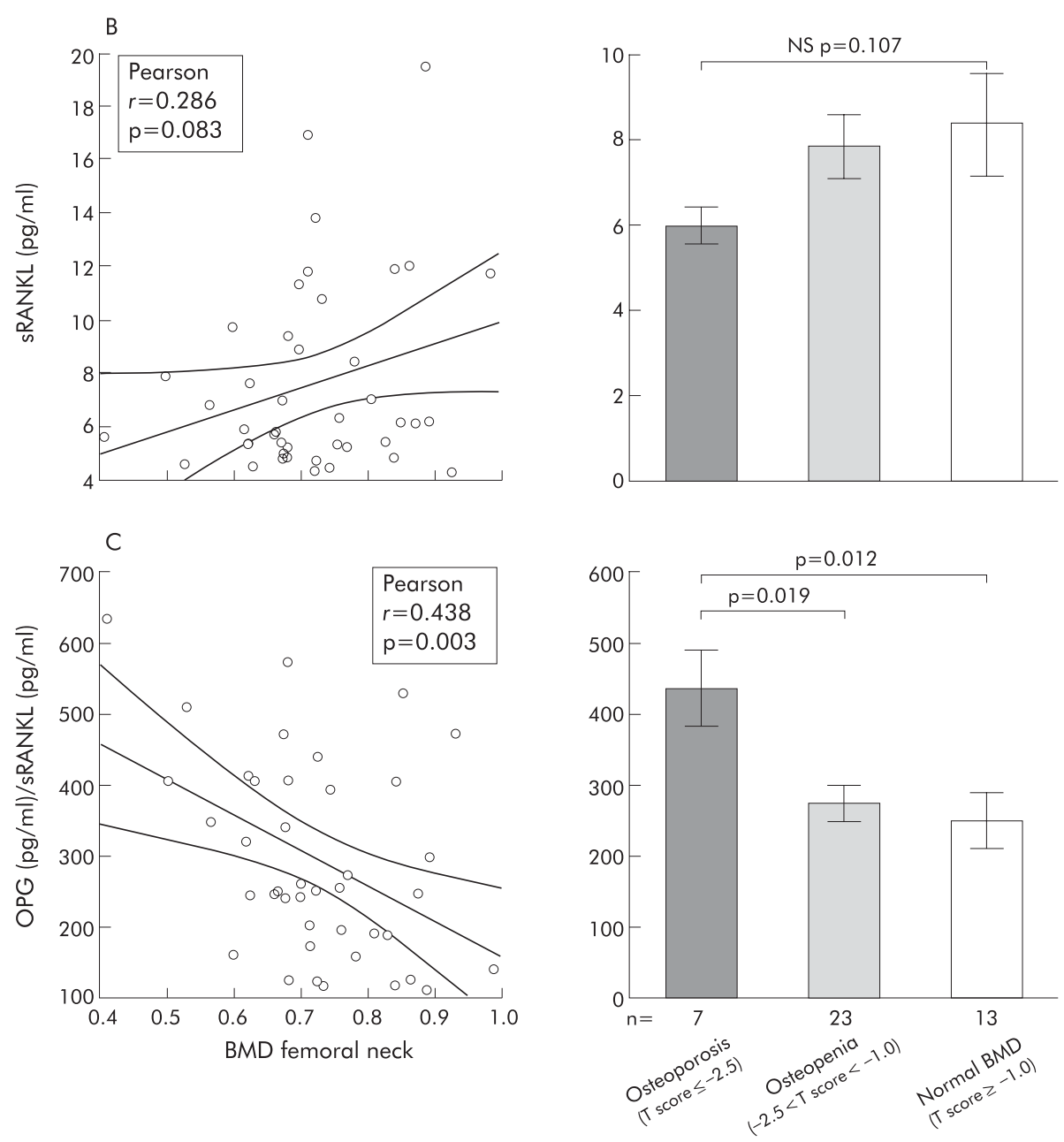

negative correlation between OPG and femoral neck (fig 2A) and lumbar spine (fig 3A) BMD was observed, although it should be noted that the correlation was not particularly strong. No such correlation was found between sRANKL plasma levels and BMD (fig 2B, 3B). Subgroup analysis in IBD patients with normal BMD ( $\mathrm{T}$ score $\geqslant-1$ ), with osteopenia $(-2.5<\mathrm{T}$ score $<-1.0)$, and with osteoporosis (T score $\leqslant-2.5)^{16}$ revealed a gradual increase in OPG plasma levels, with a statistically significant increase observed in osteoporotic patients (fig 2A, 3A). Again, no such association was found for sRANKL (fig 2B, 3B). Indeed, sRANKL levels tended to decrease with the level of bone loss although the decline did not reach statistical significance (fig 2B, 3B).

As might be inferred from the aforementioned data, analysis based on OPG/SRANKL levels showed a gradual increase in the ratio from controls to osteopenic to osteoporotic IBD patients, as demonstrated for femoral neck (fig 2C) and lumbar spine (fig 3C) BMD.

\section{Colonic explant cultures from inflamed IBD regions release large amounts of OPG}

To gain insight into whether the intestine might indeed be a source of sRANKL and OPG and therefore could in 

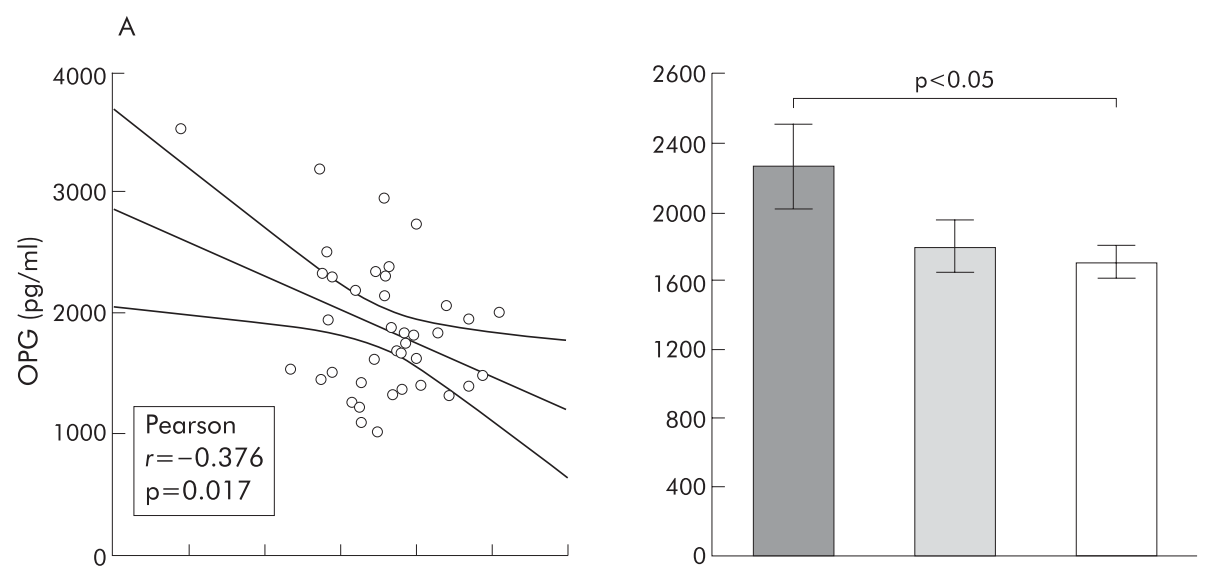

Figure 3 Osteoprotegerin (OPG) and soluble receptor activator of nuclear factor kappa B (sRANKL) plasma levels and bone mineral density (BMD) in inflammatory bowel disease (IBD) patients. Left panels show the correlation of OPG levels (A), sRANKL plasma levels (B), and the OPG/ sRANKL ratio $(C)$ with lumbar spine $B M D$. Right panels show analysis of OPG, sRANKL plasma levels, and the OPG/sRANKL ratio in IBD patients classified as normal, osteopenic, and osteoporotic according to their BMD.
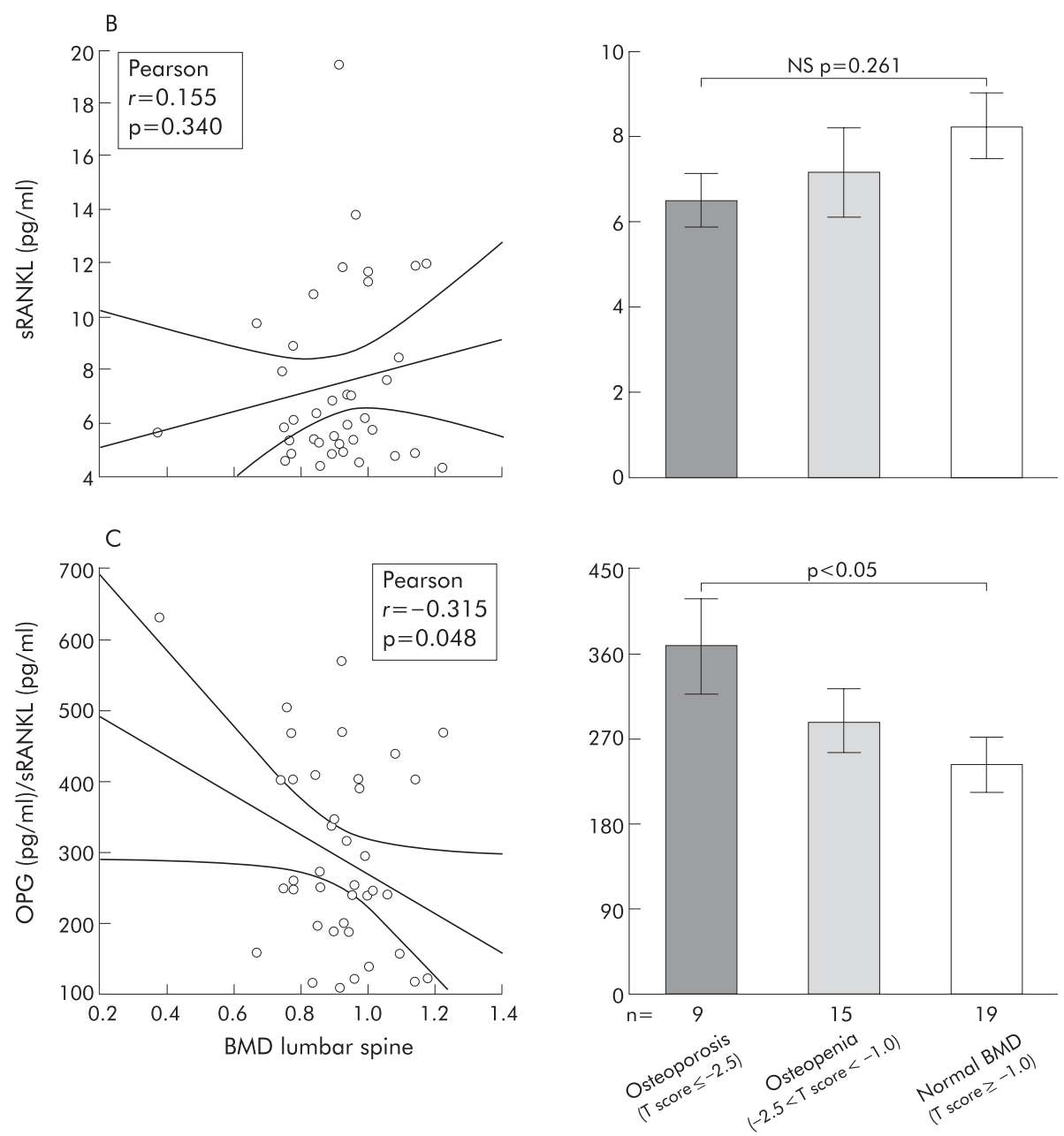

principal account for the increased plasma OPG levels observed, we obtained CEC. CEC are a useful method of determining cytokine secretion in tissue as ex vivo cultured colonic specimens are likely to reflect the in vivo situation and provide this information without the bias introduced by studying only a particular cell population or by in vitro stimulation. As shown in fig 4A, a 3.4-fold increase in OPG release was noted in specimens derived from inflamed tissue of $\mathrm{CD}$ patients compared with healthy controls while OPG release from non-involved $C D$ specimens was essentially identical to healthy controls (fig 4A). With regard to UC, OPG release from involved specimens was on average increased 3.8-fold (showing wide variation in the levels detected) while non-involved regions showed OPG levels comparable with healthy controls as well as non-involved CD (fig 4A). Low amounts of sRANKL were released irrespective of disease state in $C D, U C$, and healthy controls (fig 4B).

\section{Relationship between OPG and sRANKL levels and IBD therapy}

We next asked whether there is an association between therapy and OPG and sRANKL levels. While no such association was found for OPG (fig 5A), IBD medication had a 

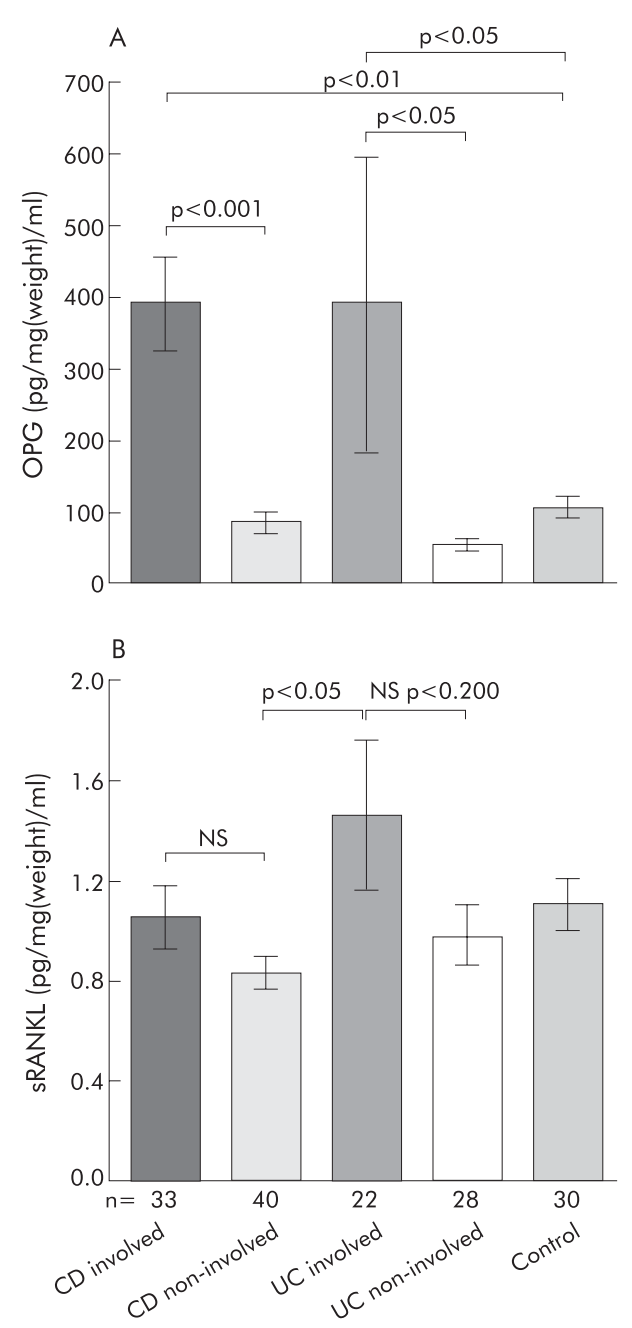

Figure 4 Osteoprotegerin (OPG) (A) and soluble receptor activator of nuclear factor kappa B (sRANKL) (B) release from colonic explant cultures. Colonic biopsies from Crohn's disease (CD), ulcerative colitis (UC), and control subjects were cultured for 24 hours and supernatants assayed for OPG and sRANKL. Data are expressed as $\mathrm{pg} / \mathrm{ml}$ normalised to the weight of the individual biopsies.

significant influence on sRANKL levels (fig 5B). Patients treated with corticosteroids or combined azathioprine and corticosteroids showed significantly lower sRANKL levels compared with IBD patients who received no specific therapy (no medication: 12.7 (1.8) pg/ml; steroid: 7.2 (0.3) pg/ml, $\mathrm{p}<0.01$; azathioprine+steroid: 7.4 (0.6) pg/ml, $\mathrm{p}<0.05$ ). sRANKL levels of patients treated with 5-aminosalicylate or azathioprine alone were lower but this difference did not reach statistical significance (fig 5B). As active and inactive IBD do not show differences in sRANKL plasma levels (data not shown), it is unlikely that differences in clinical activity scores between the various treatment groups could explain the lower sRANKL plasma levels in steroid treated patients. No significant changes associated with medication were found for the OPG/sRANKL ratio (fig 5C).

Immunohistochemical localisation of OPG and RANKL in the mucosa

While few $\mathrm{OPG}^{+}$cells were found in control mucosa, we observed abundant numbers of OPG expressing cells in colonic tissue sections derived from $\mathrm{CD}$ and $\mathrm{UC}$ patients (fig 6). While those few $\mathrm{OPG}^{+}$cells in control subjects showed relatively faint staining, OPG staining in $\mathrm{CD}$ and UC samples
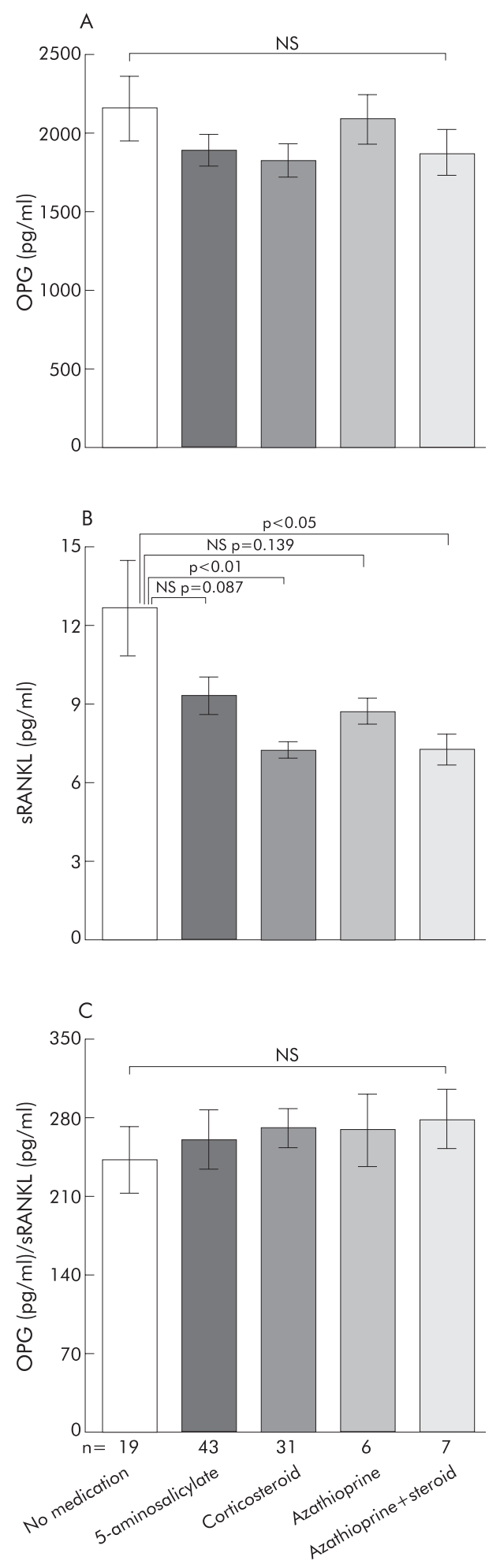

Figure 5 Association of inflammatory bowel disease (IBD) medication with osteoprotegerin (OPG) (A) and soluble receptor activator of nuclear factor kappa B (sRANKL) (B) plasma levels. Plasma levels of OPG, sRANKL, and the OPG/sRANKL ratio (C) were analysed with respect to current IBD associated medication.

was particularly bright in various cell types, including epithelial cells (fig 6). $\mathrm{OPG}^{+}$cells were especially present in lymphoid aggregates, and notably in the lamina muscularis, the latter being completely absent in control subjects (data not shown). In control subjects, $\mathrm{RANKL}^{+}$cells were 


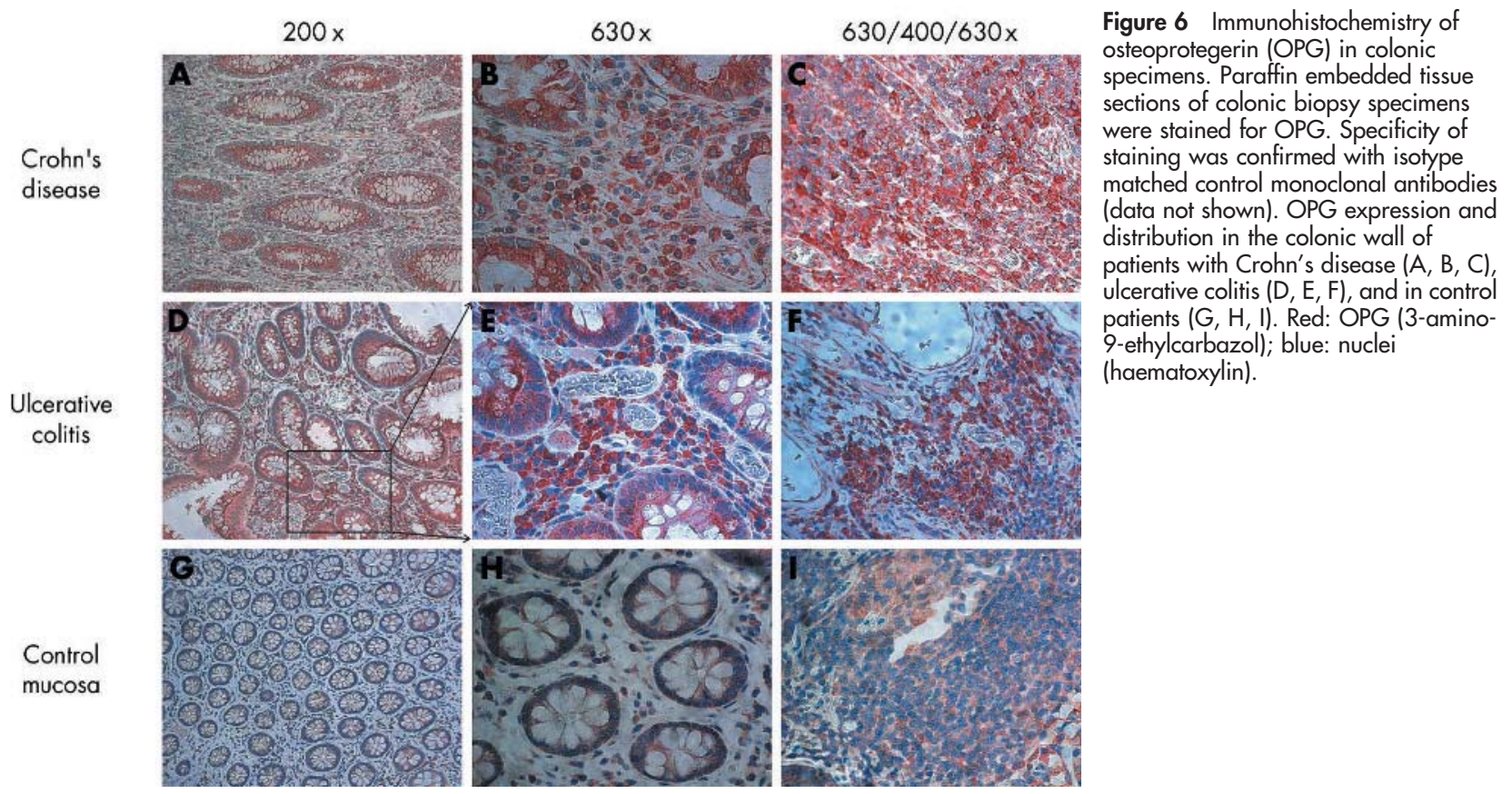

predominantly found in the submucosa while they were also present in between crypts in CD and UC (fig 7). Furthermore, $\mathrm{RANKL}^{+}$cells were found in the lamina muscularis in CD whereas this finding was less pronounced in UC and absent in healthy controls (fig 7).

\section{Identification of cellular sources of OPG and RANKL in the colonic mucosa}

To gain insight into the cellular sources of OPG and RANKL in IBD mucosa, we performed immunofluorescence double staining for CD3, MHC II, CD163, and DC-LAMP. As depicted in fig $8(\mathrm{~A}, \mathrm{~B}, \mathrm{C})$, OPG predominantly colocalised with $\mathrm{MHC}$ II $^{\text {bright }}$, CD 163, and DC-LAMP, suggesting macrophages and dendritic cells as primary sources. In contrast, RANKL

primarily colocalised with CD3, suggesting $\mathrm{T}$ lymphocytes as an important source, but was also detected on some MHC $\mathrm{II}^{+}$cells (fig 8D, E, F).

\section{DISCUSSION}

In this study we have described alterations in the RANKL/ OPG system in IBD and its relationship to decreased BMD in these patients. We demonstrated increased plasma levels of OPG as well as increased release from the inflamed colon in IBD, suggesting the colonic mucosa as a source of circulating OPG in IBD. The relative contribution of mucosa derived OPG and sRANKL to their corresponding serum levels was difficult to estimate in this human study. Furthermore, OPG plasma levels were inversely correlated with BMD (that is,

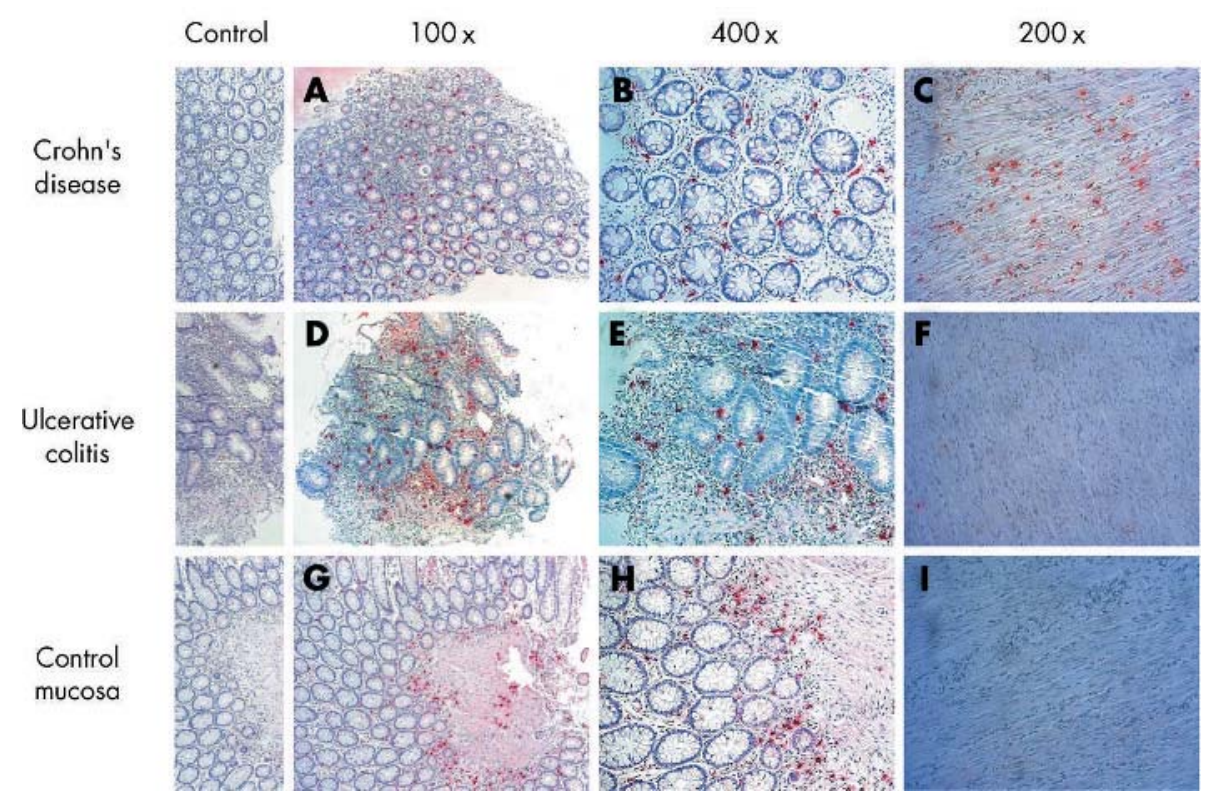

Figure 7 Immunohistochemistry of receptor activator of nuclear factor kappa B (RANKL) in colonic biopsy specimens and surgical resections. RANKL expression differed in patients with Crohn's disease (CD) (A, B, C), ulcerative colitis (UC) (D, E, F), and control patients $(G, H$, I). In control patients, RANKL cells were predominantly found in the submucosa $(G, H)$ whereas in $C D(A, B)$ and $U C(D$, $\mathrm{E}), \mathrm{RANKL}^{+}$cells were additionally present between the crypts. Notably, numerous $\mathrm{RANKL}^{+}$cells were localised in the lamina muscularis of $C D$ patients (C). However, RANKL ${ }^{+}$cells were rare in UC (F) and absent in the lamina muscularis of control patients (I). Controls were performed using isotype matched control monoclonal antibodies (left panel). Red: RANKL (Vector Red); blue: nuclei (haematoxylin). 


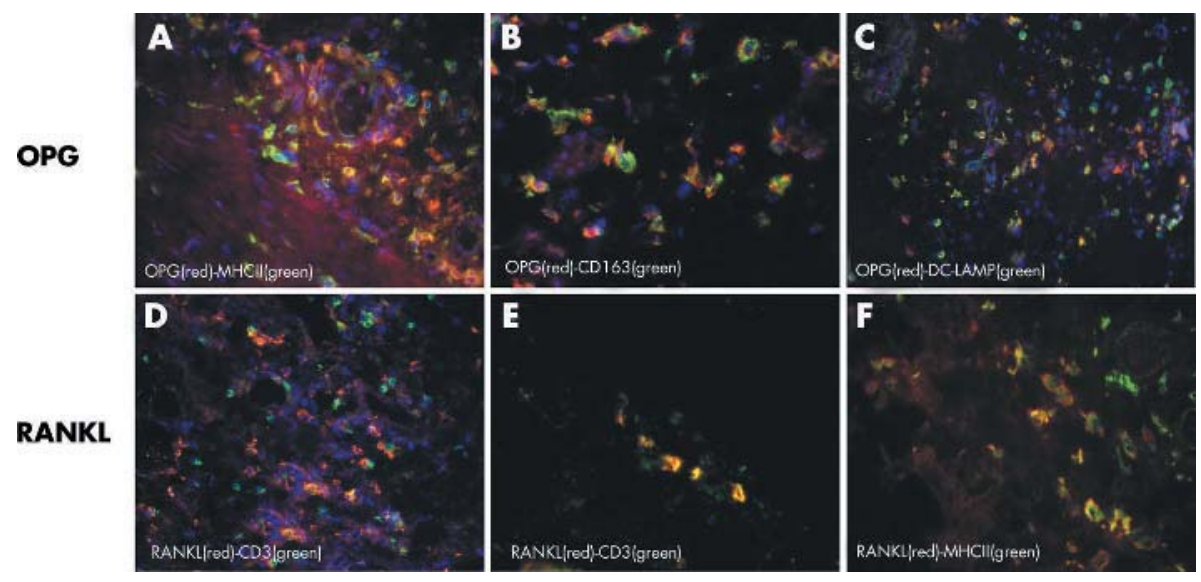

Figure 8 Cellular localisation of osteoprotegerin (OPG) and receptor activator of nuclear factor kappa B (RANKL) in colonic mucosa. OPG (A, B, C; red) and RANKL (D, E, F; red) expression in colonic tissue specimens was analysed by double immunofluorescent microscopy to identify cell types secreting these molecules. Anti-MHC II (DC, macrophages, B cells), anti-CD163 (macrophages), anti-DC-LAMP (activated DC), and anti-CD3 (T lymphocytes) are shown in green. Double staining is represented by yellow (green+red). Cell nuclei are shown in blue. Specificity of staining was confirmed by isotype matched irrelevant monoclonal antibodies for all antibodies used (data not shown). OPG primarily colocalised with MHC Iligh, CD163, and DC-LAMP. RANKL predominantly colocalised with CD3, and in smaller numbers with MHC II. Magnification: A, E, 400×; B, F, 630×; C', $D, 200 \times$.

osteoporotic IBD patients showed substantial elevation in circulating $\mathrm{OPG}$ ). On the other hand, we did not observe any change in sRANKL plasma levels or sRANKL release from CEC in IBD, although we noted increased numbers of $\mathrm{RANKL}^{+}$cells, particularly in the lamina muscularis of IBD patients.

At first glance, an increased level of the decoy receptor (OPG) with virtually no change in the osteoclastogenic agonist (sRANKL) in IBD strongly argues against a role for the RANKL system in the development of bone loss and osteoporosis in IBD. Indeed, the opposite could be true. This view is supported by the recent demonstration of mechanisms of bone loss via the RANK/RANKL system in the IL-2 deficient mouse model of colitis, which may be a valuable model for bone loss in IBD. ${ }^{10}$ Colitic IL- $2^{-/-}$mice develop substantial osteopenia with increasing age, with bone loss detectable at the onset of colitis at the age of four weeks. ${ }^{10}$ Development of bone loss in this model is clearly dependent on sRANKL release from activated T cells. ${ }^{10}$ Notably, elevated sRANKL serum levels are only observed at an early stage at the onset of bone loss and colitis, and decline rapidly to normal levels by week $9 .{ }^{10}$ In contrast, OPG levels start to substantially increase later, without ever reaching a plateau until the end of the observation period. ${ }^{10}$ This may explain why we "only" observed normal levels of sRANKL and an increase in OPG levels in our human study, which could well be interpreted as an indication of the involvement of this system in IBD associated bone loss.

As in the mouse colitis model, increased OPG levels may represent a continuing homeostatic response, attempting to reverse established osteopenia and RANKL driven osteoclastogenesis, thus maintaining normal bone mass. This view is supported by the gradual increase in OPG levels in osteopenic and osteoporotic IBD patients, as shown in our study. However, longitudinal studies of OPG/RANKL and BMD are required to validate this model and to assess the relative contribution of inflammation to bone loss in human disease.

Some of the osteoclastogenic factors released from the IBD mucosa (for example, IL-1, IL-6, TNF- $\alpha$ ) are thought to function indirectly via specific receptors on stromal osteoblastic cells to enhance RANKL expression. ${ }^{1718}$ Notably, IL- $1 \beta$ and TNF- $\alpha$ concomitantly increase OPG expression by stromal osteoblasts. ${ }^{18} 19$ Thus cytokines known to perpetuate colitis simultaneously activate the RANKL/OPG system. Beyond that, RANK/RANKL interaction could also promote colitis per se: ligation of dendritic cell expressed RANK with RANKL represents a potent maturation signal for these antigen presenting cells and, indeed, treatment of IL-2 deficient colitic mice with OPG-Fc fusion protein ameliorated colitis, apparently via a substantial reduction in dendritic cells with a mature phenotype in the colonic mucosa. ${ }^{10}$ With regard to the colonic source of OPG in IBD, we identified cells coexpressing macrophage and dendritic cell markers while sRANKL apparently derived predominantly from T lymphocytes.

Activation of the RANKL/OPG system has already been described in several other chronic inflammatory conditions. ${ }^{12}$ Soluble RANKL as well as OPG levels are elevated in rheumatoid arthritis, while high OPG and decreased sRANKL levels have been reported in primary biliary cirrhosis. ${ }^{20}$ Bone loss in IBD is apparently multifactorial in origin (recently reviewed by Bernstein and Leslie ${ }^{9}$ ). Although it is virtually impossible in the human system to precisely determine the relative contribution of the various factors that lead to bone loss in IBD, our human data interpreted in the context of the IL-2 ${ }^{-/-}$animal model suggest that the RANKL/OPG system could be one factor indirectly associating inflammation with bone loss in IBD. Of particular note, glucocorticoid total dose was inversely associated with BMD in many studies of IBD, ${ }^{21}{ }^{22}$ but not all. ${ }^{23}$ Current glucocorticoid use has been reported to show both no effect on $\mathrm{BMD}^{23}$ and diminished BMD. ${ }^{24}$ Against common belief, it is thus still uncertain if an apparent glucocorticoid effect results from the medication itself or is simply a marker of more severe IBD. ${ }^{9}$ In this regard, it is interesting to note that we observed decreased levels of sRANKL in steroid treated IBD patients while there was no obvious alteration in OPG plasma levels in such patients.

In conclusion, we have provided evidence that the RANKL/ OPG system is altered in IBD and further studies on its pharmacological manipulation in IBD is warranted.

\section{Authors' affiliations}

A R Moschen*, A Kaser*, B Enrich, O Ludwiczek, A M Wolf, H Tilg, Division of Gastroenterology and Hepatology, Department of Medicine, University Hospital Innsbruck, Innsbruck, Austria 
M Gabriel, Department of Nuclear Medicine, University Hospital Innsbruck, Innsbruck, Austria

P Obrist, Department of Pathology, University Hospital Innsbruck, Innsbruck, Austria

*A R Moschen and A Kaser contributed equally to this work.

Conflict of interest: None declared.

\section{REFERENCES}

1 Podolsky DK. Inflammatory bowel disease. N Engl J Med 2002;347:417-29.

2 Fiocchi C. Inflammatory bowel disease: etiology and pathogenesis. Gastroenterology 1998;115:182-205.

3 Strober W, Fuss IJ, Blumberg RS. The immunology of mucosal models of inflammation. Annu Rev Immunol 2002;20:495-549.

4 Kirchgatterer A, Wenzl HH, Aschl G, et al. Examination, prevention and treatment of osteoporosis in patients with inflammatory bowel disease: recommendations and reality. Acta Med Austriaca 2002;29:120-3.

5 Lamb EJ, Wong T, Smith DJ, et al. Metabolic bone disease is present at diagnosis in patients with inflammatory bowel disease. Aliment Pharmacol Ther 2002; 16:1895-902.

6 Bernstein CN, Leslie WD, Leboff MS. AGA technical review on osteoporosis in gastrointestinal diseases. Gastroenterology 2003;124:795-841.

7 American Gastroenterological Association medical position statement: guidelines on osteoporosis in gastrointestinal diseases. Gastroenterology 2003; 124:791-4

8 de Jong DJ, Corstens FH, Mannaerts L, et al. Corticosteroid-induced osteoporosis: does it occur in patients with Crohn's disease? Am J Gastroenterol 2002;97:2011-15.

9 Bernstein CN, Leslie WD. The pathophysiology of bone disease in gastrointestinal disease. Eur J Gastroenterol Hepatol 2003;15:857-64

10 Ashcroft AJ, Cruickshank SM, Croucher PI, et al. Colonic dendritic cells intestinal inflammation, and T cell-mediated bone destruction are modulated by recombinant osteoprotegerin. Immunity 2003;19:849-61.

11 Locksley RM, Killeen N, Lenardo MJ. The TNF and TNF receptor superfamilies: integrating mammalian biology. Cell 2001;104:487-501.
12 Theill LE, Boyle WJ, Penninger JM. RANK-L and RANK: T cells, bone loss, and mammalian evolution. Annu Rev Immunol 2002;20:795-823.

13 Lacey DL, Timms E, Tan HL, et al. Osteoprotegerin ligand is a cytokine that regulates osteoclast differentiation and activation. Cell 1998;93:165-76.

14 Simonet WS, Lacey DL, Dunstan CR, et al. Osteoprotegerin: a novel secreted protein involved in the regulation of bone density. Cell 1997;89:309-19.

15 Schreiber S, Heinig T, Thiele HG, et al. Immunoregulatory role of interleukin 10 in patients with inflammatory bowel disease. Gastroenterology 1995: 108:1434-44.

16 Word Health Organization Study Group on Assessment of Fracture Risk and its Application to Screening for Postmenopausal Osteoporosis. Assessment of fracture risk and its application to screening for postmenopausal osteoporosis. Geneva: World Health Organization, 1994.

17 Kong YY, Feige U, Sarosi I, et al. Activated T cells regulate bone loss and joint destruction in adjuvant arthritis through osteoprotegerin ligand. Nature 1999:402:304-9.

18 Romas E, Gillespie MT, Martin TJ. Involvement of receptor activator of NFkappaB ligand and tumor necrosis factor-alpha in bone destruction in rheumatoid arthritis. Bone 2002;30:340-6.

19 Hofbauer LC, Lacey DL, Dunstan CR, et al. Interleukin-1 beta and tumor necrosis factor-alpha, but not interleukin-6, stimulate osteoprotegerin ligand gene expression in human osteoblastic cells. Bone 1999;25:255-9.

20 Szalay F, Hegedus D, Lakatos PL, et al. High serum osteoprotegerin and low RANKL in primary biliary cirrhosis. J Hepatol 2003;38:395-400.

21 Abitbol V, Roux C, Chaussade S, et al. Metabolic bone assessment in patients with inflammatory bowel disease. Gastroenterology 1995;108:417-22.

22 Silvennoinen JA, Karttunen TJ, Niemela SE, et al. A controlled study of bone mineral density in patients with inflammatory bowel disease. Gut 1995; $37: 71-6$

23 Bjarnason I, Macpherson A, Mackintosh C, et al. Reduced bone density in patients with inflammatory bowel disease. Gut 1997;40:228-33.

24 Robinson RJ, al Azzawi F, labal SJ, et al. Osteoporosis and determinants of bone density in patients with Crohn's disease. Dig Dis Sci 1998:43:2500-6.

25 Best WR, Becktel JM, Singleton JW, et al. Development of a Crohn's disease activity index. National Cooperative Crohn's Disease Study. Gastroenterology 1976;70:439-44.

26 Rachmilewitz D. Coated mesalazine (5-aminosalicylic acid) versus sulphasalazine in the treatment of active ulcerative colitis: a randomised trial. BMJ 1989:298:82-6.

\section{EDITOR'S QUIZ: GI SNAPSHOT}

\section{Answer}

From question on page 454

Abdominal computed tomography revealed gastric pneumatosis with pneumoperitoneum and complete thrombosis of the coeliac trunk. Upper endoscopy showed necrosis of the lesser curvature reaching the duodenum, diffuse erythema and swelling of the remaining gastric mucosa, and a prepyloric $1 \mathrm{~cm}$ wide gastric ulcer. Purulent sterile liquid surrounded the stomach at laparotomy. The great tuberosity of the stomach was necrotic with diffuse surface necrosis of the remaining stomach and the right and sigmoid colon. The small intestine was also ischaemic with diffuse petechiae on its surface. Extended thrombosis of the left femoral artery precluded clearing of the obstruction with a Fogarty catheter. At this point, no procedure was deemed possible and the patient died 12 hours later.

Gastric pneumatosis is not a disease but a finding that should be investigated based on the patient's clinical situation, and on his past and presenting history. These situations include: (1) gastric emphysema (gastric outlet syndrome, gastric carcinoma, intestinal volvulus, small bowel obstruction, forceful vomiting, and gall stones); (2) emphysematous gastritis (Escherichia coli, Enterobacter, Clostridium species, Pseudomonas aeruginosa, Bacillus species, Aerobacter aerogenes, Staphylococcus aureus, caustic or corrosive substances, alcohol intoxication, ischaemia, infarction, previous gastrointestinal surgery, strongyloidosis, diabetes, and immunosuppression); and (3) pneumatosis cystoides intestinalis (neoplastic, infectious, chemicals, and mechanical).

doi: 10.1136/gut.2004.049494 